\title{
Mucosal CD30-positive T-cell lymphoproliferations of the head and neck show a clinicopathologic spectrum similar to cutaneous CD30-positive T-cell lymphoproliferative disorders
}

\author{
Andrew P Sciallis ${ }^{1}$, Mark E Law ${ }^{1}$, David J Inwards ${ }^{2}$, Rebecca F McClure ${ }^{1}$, \\ William R Macon ${ }^{1}$, Paul J Kurtin ${ }^{1}$, Ahmet Dogan ${ }^{1}$ and Andrew L Feldman ${ }^{1}$ \\ ${ }^{1}$ Department of Laboratory Medicine and Pathology, Mayo Clinic, Rochester, MN, USA and \\ ${ }^{2}$ Department of Internal Medicine, Mayo Clinic, Rochester, MN, USA
}

\begin{abstract}
CD30-positive T-cell lymphoproliferative disorders are classified as cutaneous (primary cutaneous anaplastic large cell lymphoma and lymphomatoid papulosis) or systemic. As extent of disease dictates prognosis and treatment, patients with skin involvement need clinical staging to determine whether systemic lymphoma also is present. Similar processes may involve mucosal sites of the head and neck, constituting a spectrum that includes both neoplasms and reactive conditions (eg, traumatic ulcerative granuloma with stromal eosinophilia). However, no standard classification exists for mucosal CD30-positive T-cell lymphoproliferations. To improve our understanding of these processes, we identified 15 such patients and examined clinical presentation, treatment and outcome, morphology, phenotype using immunohistochemistry, and genetics using gene rearrangement studies and fluorescence in situ hybridization. The 15 patients (11 M, $4 \mathrm{~F}$; mean age, 57 years) had disease involving the oral cavity/lip/tongue (9), orbit/conjunctiva (3) or nasal cavity/sinuses (3). Of 14 patients with staging data, 7 had mucosal disease only; 2 had mucocutaneous disease; and 5 had systemic anaplastic large cell lymphoma. Patients with mucosal or mucocutaneous disease only had a favorable prognosis and none developed systemic spread (follow-up, 4-93 months). Three of five patients with systemic disease died of lymphoma after 1-48 months. Morphologic and phenotypic features were similar regardless of extent of disease. One anaplastic lymphoma kinase-positive case was associated with systemic disease. Two cases had rearrangements of the DUSP22-IRF4 locus on chromosome 6p25.3, seen most frequently in primary cutaneous anaplastic large cell lymphoma. Our findings suggest mucosal CD30-positive T-cell lymphoproliferations share features with cutaneous CD30-positive T-cell lymphoproliferative disorders, and require clinical staging for stratification into primary and secondary types. Primary cases have clinicopathologic features closer to primary cutaneous disease than to systemic anaplastic large cell lymphoma, including indolent clinical behavior. Understanding the spectrum of mucosal CD30-positive T-cell lymphoproliferations is important to avoid possible overtreatment resulting from a diagnosis of overt T-cell lymphoma. Modern Pathology (2012) 25, 983-992; doi:10.1038/modpathol.2012.38; published online 2 March 2012
\end{abstract}

Keywords: anaplastic large cell lymphoma; CD30; lymphomatoid papulosis; mucosal lymphoma; T-cell lymphoma; traumatic ulcerative granuloma with stromal eosinophilia; traumatic eosinophilic granuloma

Correspondence: Dr AL Feldman, MD, Department of Laboratory Medicine and Pathology, Mayo Clinic, 200 First Street SW, Rochester, MN 55905, USA.

E-mail: feldman.andrew@mayo.edu

Received 12 December 2011; revised 5 January 2012; accepted 5 January 2012; published online 2 March 2012
The World Health Organization (WHO) classification of hematopoietic neoplasms uses a combination of morphologic, phenotypic, genetic and clinical features to separate distinct disease entities. ${ }^{1}$ Among clinical features, anatomic site is of particular importance in subclassifying T-cell neoplasms. One diagnostically challenging area is the classification 
of cutaneous CD30-positive T-cell lymphoproliferative disorders, which relies heavily on clinical presentation and staging to stratify cases into primary and secondary types. ${ }^{2,3}$ This stratification has important prognostic and therapeutic implications. Primary cutaneous anaplastic large cell lymphoma and lymphomatoid papulosis require only local therapy and have 5-year overall survival rates of $90 \%$ or higher., ${ }^{4,5}$ Secondary cutaneous involvement by systemic anaplastic lymphoma kinase (ALK)-negative anaplastic large cell lymphoma has overlapping morphologic and phenotypic features but is treated with systemic combination chemotherapy and has a 5 -year overall survival rate of only $49 \%{ }^{5}$

CD30-positive T-cell lymphoproliferations also may present at mucosal sites in the head and neck, particularly the oral mucosa, but no stratification system analogous to that used for cutaneous involvement exists to classify these lesions. Terminology to describe mucosal lesions with overlapping morphologic and phenotypic features has ranged from reactive (eg, traumatic eosinophilic granuloma or traumatic ulcerative granuloma with stromal eosinophilia) to malignant (including ALK-negative anaplastic large cell lymphoma). In some cases, a reactive designation might lead to insufficient evaluation for extramucosal sites of disease, while an overtly malignant diagnosis in cases with localized disease could lead to systemic chemotherapy that might be unnecessary.

To help improve our understanding of mucosal CD30-positive T-cell lymphoproliferations of the head and neck, we reviewed our institutional experience with lesions within this spectrum based on their morphologic, phenotypic, genetic and clinical features. Our findings in 15 patients indicate CD30positive T-cell lymphoproliferations limited to mucosal sites or with a mucocutaneous pattern of distribution have clinicopathologic features closer to primary cutaneous CD30-positive T-cell lymphoproliferative disorders than to systemic anaplastic large cell lymphoma, including indolent clinical behavior. However, mucosal lesions with similar pathologic features also were seen in patients with systemic anaplastic large cell lymphoma, emphasizing the importance of clinical staging. These findings have important implications for the nomenclature and clinical management of mucosal CD30-positive T-cell lymphoproliferations.

\section{Patients and methods}

\section{Patients}

We searched the Anatomic Pathology archive of Mayo Clinic (Rochester, MN, USA) for cases of CD30-positive T-cell lymphoproliferations involving mucosal sites in the head and neck, including upper aerodigestive tract and conjunctiva/orbit (the last based in part on the inclusion of orbit as a mucosaassociated site in marginal zone B-cell lymphomas of mucosa-associated lymphoid tissue). All cases had phenotypic and/or genetic evidence of T-cell lineage and expressed CD 30 in $>75 \%$ of cells (based on criteria established for primary cutaneous CD30positive T-cell lymphoproliferative disorders). ${ }^{3}$ We identified 15 patients with pathology material dating from 1994 to 2011 that met the above criteria. We recorded clinical data including age, sex, mucosal site of involvement, clinical stage, presence or absence of involvement of other anatomic sites, administration of chemotherapy, duration of follow-up and status at most recent follow-up. The study was approved by the Mayo Clinic Institutional Review Board and the Mayo Clinic Biospecimens Committee.

\section{Morphologic and Immunophenotypic Studies}

Hematoxylin and eosin-stained sections were prepared from B5 and/or formalin-fixed material from all available biopsies for each patient. Low-power architecture, cell morphology, the presence or absence of accompanying eosinophils, and the presence or absence of mucosal ulceration were recorded. Immunohistochemistry was performed as described previously $^{6}$ using antibodies against the B-cell antigen CD20; the T-cell antigens CD2, CD3, CD4, CD5, CD7 and CD8; CD30; and ALK (Table 1) using either the DAKO (Carpenteria, CA, USA) Universal Staining System or the Ventana (Tucson, AZ, USA) BenchMark ULTRA Platform.

\section{Genetic Studies}

T-cell receptor gene rearrangement studies were performed as described previously ${ }^{7}$ in all cases for which appropriate material was available. Briefly, DNA was extracted from paraffin-embedded tissue and polymerase chain reaction (PCR) was performed on a Hybaid (Franklin, MA, USA) thermal cycler using primers specific for segments of the VDJ region of the T-cell receptor beta locus (TRB@), the VJ region of the T-cell receptor gamma locus (TRG@) and a control template to monitor the quality and extent of DNA amplification. PCR product fragments

Table 1 Antibodies used for immunohistochemistry

\begin{tabular}{|c|c|c|c|}
\hline Antigen & Clone & Dilution & Source \\
\hline ALK & ALK1 & $1: 25$ & Dako (Carpinteria, CA, USA) \\
\hline CD2 & AB75 & $1: 40$ & Novocastra (Newcastle-upon-Tyne, UK) \\
\hline CD3 & PS1 & $1: 100$ & Novocastra \\
\hline CD4 & 4B12 & $1: 20$ & Novocastra \\
\hline CD5 & $4 \mathrm{C} 7$ & $1: 25$ & Novocastra \\
\hline CD7 & LP15 & $1: 100$ & Novocastra \\
\hline CD8 & $\mathrm{C} 8 / 144$ & $1: 50$ & Dako \\
\hline CD20 & L26 & $1: 200$ & Dako \\
\hline CD30 & Ber-H2 & $1: 20$ & Dako \\
\hline
\end{tabular}


were analyzed on an ABI 3130xl Genetic Analyzer (Applied Biosystems, Foster City, CA, USA).

Fluorescence in situ hybridization (FISH) was performed on all cases for which adequate material was available using a breakapart probe for the DUSP22-IRF4 locus on 6p25.3, as described previously. ${ }^{8}$ Briefly, DNA from bacterial artificial chromosomes CTD-2308G5 (telomeric) or RP11164H16 (centromeric) was labeled with Texas ReddUTP (Molecular Probes, Invitrogen) or SpectrumGreen-dUTP (Abbott Molecular, Des Plaines, IL, USA), respectively. Paraffin-embedded tissue sections were digested in $0.4 \%$ pepsin, hybridized with probe, washed, counterstained with $4^{\prime}, 6$-diamidino2-phenylindole dihydrochloride and analyzed by a microscopist (MEL) using parameters and normal thresholds described previously. ${ }^{8}$

\section{Results}

\section{Clinical Characteristics}

Clinical characteristics of the 15 patients in the study are summarized in Table 2. There were 11 males and 4 females (M:F ratio, 2.8:1) with a mean age of 57 years (range, 5-84 years). The majority of our patients $(9 / 15,60 \%)$ presented with disease in the oral cavity, mucosal surface of the lip or tongue, with the remainder presenting with lesions involving the orbit/conjunctiva $(3 / 15,20 \%)$ or the nasal cavity/sinuses $(3 / 15,20 \%)$. No patient had a known history of mucosal trauma. Staging, treatment and follow-up data were available in 14 of 15 patients $(93 \%)$. In 7 of these 14 patients (50\%), disease was limited to the primary mucosal site of involvement. Two patients $(14 \%)$ had both mucosal and cutaneous disease (primary cutaneous anaplastic large cell lymphoma or mycosis fungoides) without involvement of other sites, and five patients (36\%) had systemic disease involving lymph nodes and/or visceral sites.

Treatment included excision of the mucosal lesion in all patients. Local external beam radiotherapy was given in 7 of 14 cases where treatment was known. Systemic chemotherapy was given in 4 of 7 localized cases, 0 of 2 mucocutaneous cases and 5 of 5 systemic cases. Median follow-up was 18 months (range, 1-93 months). At last follow-up, all seven patients with disease limited to the mucosa either were alive without evidence of disease or had died free of disease. Of the two patients with mucocutaneous disease, one was alive at last follow-up and one died of unrelated causes; both had persistent skin lesions but neither had recurrence of mucosal disease. Of the five patients with systemic disease, three died of disease after 1-48 months and the other two were alive without evidence of disease at last follow-up (21 and 82 months). Staging, treatment and follow-up data were not available in one patient with an orbital lesion (case 15).

\section{Pathologic Characteristics}

Pathologic characteristics of the 15 cases are summarized in Table 3. Morphologically, all cases demonstrated an infiltrate of large atypical lymphoid cells, some demonstrating histologic features resembling the so-called 'hallmark' cells described

Table 2 Clinical characteristics of mucosal CD30-positive T-cell lymphoproliferations

\begin{tabular}{|c|c|c|c|c|c|c|c|}
\hline Case & Age/sex & Mucosal site & Other sites & $X R T$ & CTX & $F / U$ (months) & Status \\
\hline \multicolumn{8}{|c|}{ Mucosal involvement only } \\
\hline 1 & $62 / \mathrm{M}$ & Nasal Cavity & None & Yes & Yes & 18 & NED \\
\hline 2 & $39 / \mathrm{F}$ & Orbit & None & No & Yes & 5 & NED \\
\hline 3 & $56 / \mathrm{F}$ & Conjunctiva & None & No & No & 10 & NED \\
\hline 4 & $81 / \mathrm{M}$ & Oral Cavity & None & Yes & No & 31 & DFD \\
\hline 5 & $79 / \mathrm{M}$ & Tongue & None & No & Yes & 93 & DFD \\
\hline 6 & $68 / \mathrm{M}$ & Tongue & None & No & Yes & 7 & NED \\
\hline 7 & $77 / \mathrm{M}$ & Lower lip & None & Yes & No & 4 & NED \\
\hline \multicolumn{8}{|c|}{ Mucosal and skin involvement } \\
\hline 8 & $73 / \mathrm{M}$ & Paranasal sinus & Skin (mycosis fungoides) & No & No & 19 & DOC \\
\hline 9 & $84 / \mathrm{M}$ & Tongue & Skin (primary cutaneous anaplastic large cell lymphoma) & Yes & No & 66 & NED \\
\hline \multicolumn{8}{|c|}{ Systemic anaplastic large cell lymphoma } \\
\hline 10 & $5 / \mathrm{M}$ & Hard palate & LN & No & Yes & 82 & NED \\
\hline 11 & $50 / \mathrm{F}$ & Upper lip & LN, skin, soft tissue & No & Yes & 21 & NED \\
\hline 12 & $78 / \mathrm{M}$ & Hard palate & Small bowel and mesentery & Yes & Yes & 48 & DOD \\
\hline 13 & $52 / \mathrm{M}$ & Oral cavity & Stomach, lung, parotid, LN & Yes & Yes & 6 & DOD \\
\hline 14 & $23 / \mathrm{M}$ & Frontal sinus & LN, soft tissue & Yes & Yes & 1 & DOD \\
\hline \multicolumn{8}{|c|}{ Staging data unavailable } \\
\hline 15 & $33 / \mathrm{F}$ & Orbit & $?$ & $?$ & $?$ & None & ? \\
\hline
\end{tabular}

Abbreviations: CTX, systemic chemotherapy; DFD, died free of disease; DOC, died of other causes; F, female; F/U, follow-up; LN, lymph node; $\mathrm{M}$, male; NED, alive with no evidence of disease; XRT, external beam radiotherapy. 
Table 3 Pathologic characteristics of mucosal CD30-positive T-cell lymphoproliferations

\begin{tabular}{|c|c|c|c|c|c|c|c|}
\hline Case & Ulcer & Eos. & T-cell antigens expressed & T-cell antigens aberrantly absent & $A L K$ & T-cell receptor PCR & 6p25.3 FISH \\
\hline \multicolumn{8}{|c|}{ Mucosal involvement only } \\
\hline 1 & + & + & $\mathrm{CD} 2$ & CD3, CD4+CD8, CD5, CD7 & - & + & + \\
\hline 2 & ? & - & CD2, CD3, CD4, CD5 & CD7 & - & + & ND \\
\hline 3 & + & - & CD2, CD3, CD4, CD5 & CD7 & - & + & - \\
\hline 4 & + & + & CD2, CD3, CD4, CD5 & CD7 & - & No amp & - \\
\hline 5 & + & ++ & $\mathrm{CD} 2, \mathrm{CD} 3, \mathrm{CD} 4$ & - & - & No amp & - \\
\hline 6 & + & ++ & $\mathrm{CD} 2, \mathrm{CD} 3, \mathrm{CD} 4$ & CD5, CD7 & - & ND & ND \\
\hline 7 & + & ++ & CD2, CD3, CD8 & CD5, CD7 & - & ND & - \\
\hline \multicolumn{8}{|c|}{ Mucosal and skin involvement } \\
\hline 8 & + & ++ & $\mathrm{CD} 2, \mathrm{CD} 4$ & CD3 & - & No amp & - \\
\hline 9 & - & - & $\mathrm{CD} 2, \mathrm{CD} 3$ & - & - & ND & - \\
\hline \multicolumn{8}{|c|}{ Systemic anaplastic large cell lymphoma } \\
\hline 10 & + & + & $\mathrm{CD} 2, \mathrm{CD} 7$ & CD3, CD5 & + & ND & - \\
\hline 11 & ? & ++ & CD2, CD3, CD4 & CD5, CD7 & - & + & - \\
\hline 12 & + & - & $\mathrm{CD} 2, \mathrm{CD} 3, \mathrm{CD} 4$ & CD5, CD7 & - & + & - \\
\hline 13 & + & ++ & $\mathrm{CD} 3, \mathrm{CD} 4$ & - & - & No amp & - \\
\hline 14 & + & - & CD2 & CD3, CD5, CD7 & - & ND & ND \\
\hline \multicolumn{8}{|c|}{ Staging data unavailable } \\
\hline 15 & $?$ & - & CD3 & CD5 & - & + & + \\
\hline
\end{tabular}

Abbreviations: ALK, anaplastic lymphoma kinase; Eos, tissue eosinophils; FISH, fluorescence in situ hybridization; ND, not done; No amp, no amplification; PCR, polymerase chain reaction.

Ulceration: +, present; -, absent; ?, could not be assessed because no epithelial surface was present in the biopsy.

Eosinophils: ++, abundant; +, occasional; -, absent.

in systemic anaplastic large cell lymphoma, with a sheet-like growth pattern seen at least focally (Figures 1b, g and 2b, d, f, h). ${ }^{9}$ Mucosal ulceration was seen in 11 of 12 cases where the biopsy contained sufficient epithelium for evaluation (Figures 1a, f and 2c, g). At least occasional eosinophils were present in 9 of the 15 cases. Marked tissue eosinophilia was seen in six cases, including five in the oral cavity and one in the paranasal sinus. Three cases with marked eosinophilia had mucosal disease only (Figure 1g), one had mucocutaneous disease and two had systemic ALK-negative anaplastic large cell lymphoma (Figures $2 \mathrm{f}$ and $\mathrm{h}$ ).

Immunophenotypically, all cases expressed at least one T-cell antigen. Of 11 cases in which expression of CD4 and CD8 was tested, 9 were positive for CD4, 1 was positive for CD8 (case 7, Figure 1h) and 1 was double-negative for CD4 and CD8. There was aberrant loss of at least one T-lineage antigen in 12 of 15 cases $(80 \%)$, most commonly CD7. Six of the seven cases with mucosal disease only had aberrant T-antigen loss. By our inclusion criteria, all cases were strongly positive for CD30 in $>75 \%$ of tumor cells. Only one case was positive for ALK (case 10). This patient was a 5-year-old male with systemic ALK-positive anaplastic large cell lymphoma. Material was available for T-cell receptor PCR in 10 cases and amplification was adequate for interpretation in 6. All showed clonal rearrangements. FISH for the DUSP22-IRF4 locus on 6p25.3 was performed in 12 of the 15 cases and demonstrated a 6p25.3 rearrangement in 2 cases, both ALK negative. One of these (case 1) was a 62-year-old male with disease limited to the nasal cavity, and the other (case 15) was a 33-year-old female with orbital disease for whom staging and follow-up information was not available.

\section{Discussion}

Mucosal anatomic sites share structural and functional characteristics with the skin, including epithelial barrier protection, a richly vascularized subepithelial surface with secretory adnexal structures, vulnerability to traumatic insult, and the presence and/or recruitment of lymphoid cells to aid in the immunologic defense against external antigens and organisms that traverse the normal barrier function. Among the B-cell lymphoproliferative disorders, skin and mucosal sites (and their adjacent structures such as orbit) share susceptibility to extranodal marginal zone lymphoma of mucosaassociated lymphoid tissue. ${ }^{10}$ We investigated the clinicopathologic features of CD30-positive T-cell lymphoproliferations arising in mucosal sites and found that mucosal CD30-positive T-cell lymphoproliferations share important features with cutaneous CD30-positive T-cell lymphoproliferative disorders. These features include: separation into primary and secondary (systemic) types; similar morphologic and phenotypic features; lack of ALK expression in primary cases; occasional rearrangements of the DUSP22-IRF4 locus on 6p25.3; and favorable outcomes in patients with primary mucosal disease. These findings have important implications for the 

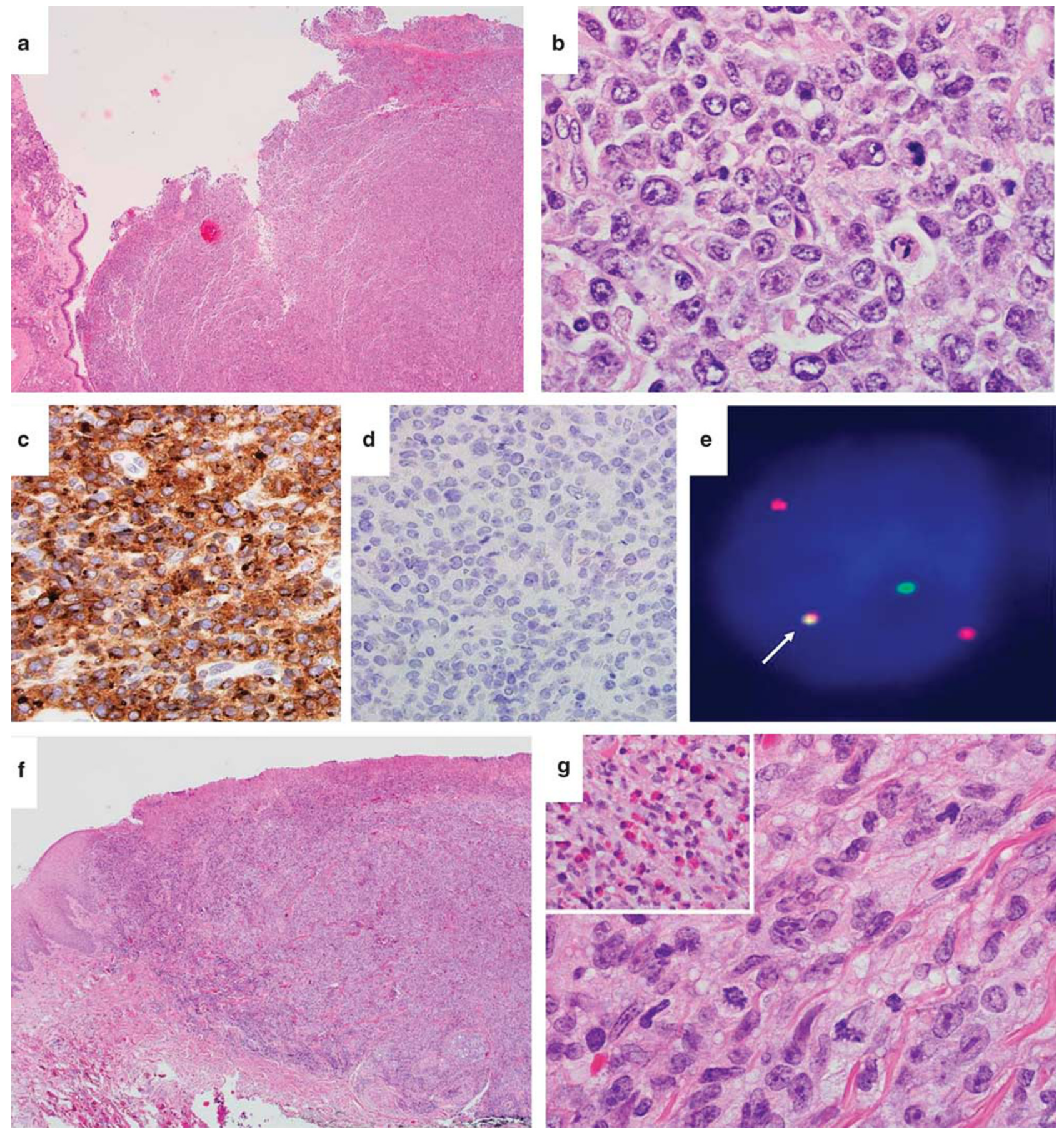

Figure 1 Primary mucosal CD30-positive T-cell lymphoproliferations without evidence of extramucosal disease. (a-e) Case 1. Biopsy of a nasal cavity mass from a 62-year-old male; staging studies were otherwise negative. An extensive submucosal infiltrate can be seen at low power (a; hematoxylin and eosin (H\&E), original magnification $\times 40)$. High power shows sheets of large atypical lymphoid cells, some with features of 'hallmark' cells $(\mathbf{b} ; \mathrm{H} \& \mathrm{E}, \times 1000)$. The atypical cells are positive for CD2 (not shown) and CD30 (c; $\times 400)$, but negative for ALK $(\mathbf{d} ; \times 400)$. Fluorescence in situ hybridization using a breakapart probe to the DUSP22-IRF4 locus on $6 \mathrm{p} 25.3$ shows separation of red and green signals as well as an intact signal (arrow); an additional red signal also is seen $(\mathbf{e} ; \times 600)$. (f-j) Case 7 . Biopsy of a mucosal lip lesion from a 77-year-old male; staging studies were otherwise negative. A vaguely nodular submucosal infiltrate with overlying ulceration can be seen at low power $(\mathbf{f} ; \mathrm{H} \& \mathrm{E}, \times 40)$. High power shows sheets of large cells $(\mathbf{g} ; \mathrm{H} \& \mathrm{E}, \times 1000)$, with some areas showing prominent collections of eosinophils (inset, $\times 400)$. The atypical cells are positive for CD8 (h, $\times 400)$ and CD30 (i, $\times 400)$; but negative for ALK $(\mathbf{j} ; \times 400)$.

classification and clinical management of mucosal CD30-positive T-cell lymphoproliferations.

Of 15 patients with mucosal CD30-positive T-cell lymphoproliferations, staging data were available in
14. Seven of these had disease limited to mucosal sites; all had favorable outcomes based on the follow-up data available (4-93 months following diagnosis), including three patients who received 

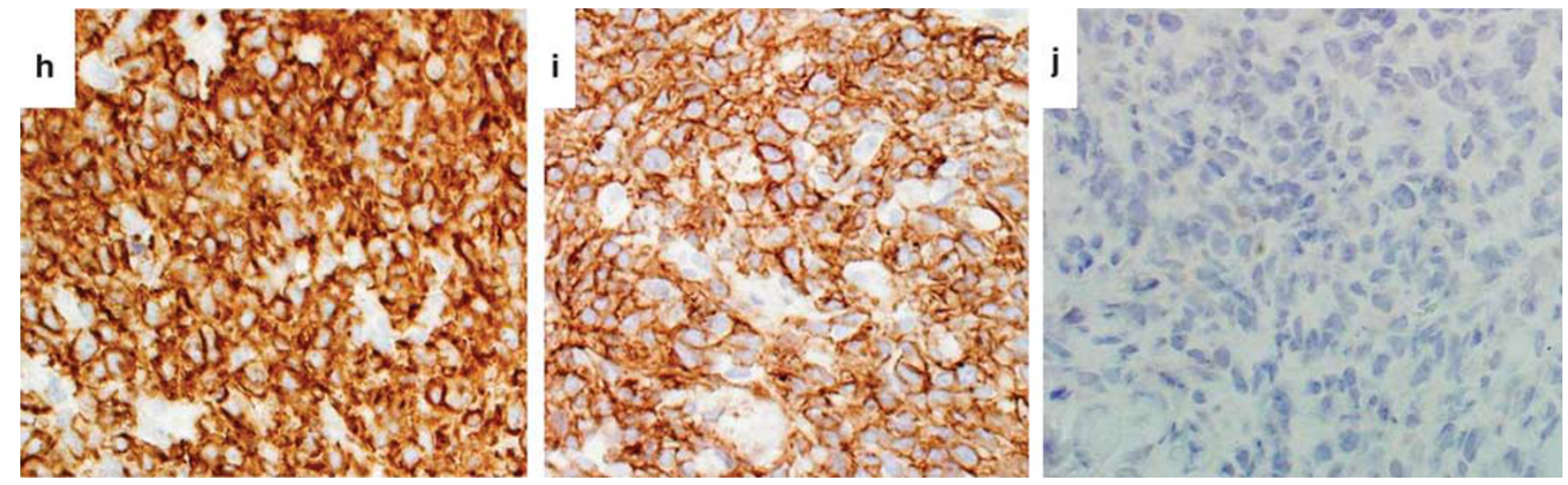

Figure 1 Continued.

no chemotherapy. Indolent CD30-positive T-cell lymphoproliferations have been reported previously in the oral cavity, especially the tongue, and variably designated traumatic ulcerative granuloma with stromal eosinophila, traumatic eosinophilic granuloma, eosinophilic ulcer of the oral mucosa and Riga (or Riga-Fede) disease. ${ }^{11-20}$ These terms connote a reactive process. ${ }^{17,19}$ Reports of seemingly similar processes have been described as lymphomatoid papulosis (in the absence of co-existing cutaneous disease $)^{21}$ or as T-cell lymphoma. ${ }^{14}$ Some investigators have used the term CD30-positive T-cell lymphoproliferative disorder and have suggested this spectrum of lesions represents the oral counterpart of cutaneous CD30-positive T-cell lymphoproliferative disorder. ${ }^{15,16,18}$ However, strict criteria for these diagnostic terms have not been defined, and only more recent studies have included examination for expression of CD30. Here, we have used the term CD30-positive T-cell lymphoproliferations as it is not certain in all cases whether these are reactive or neoplastic processes. Common features include localized disease, indolent course (sometimes including spontaneous regression with or without subsequent recurrence), ulceration, the presence of large CD30-positive T cells with variable cytologic atypia and numerous admixed eosinophils. Clonal T-cell receptor gene rearrangements may be present. ${ }^{19}$ These general features fit our cases $4-7$, although eosinophila was not prominent in case 4 .

We also report three patients (cases 1-3) with disease localized to mucosal sites outside the oral cavity, including the nasal cavity, orbit and conjunctiva. Ulceration was present when it could be assessed, but prominent tissue eosinophilia was absent. To date, none of these three patients has experienced recurrence, suggesting clinical behavior similar to the patients with localized oral disease. To our knowledge, localized CD30-positive T-cell lymphoproliferations of the nasal cavity, sinuses and conjunctiva (without eyelid involvement) have not been reported. A CD30-positive T-cell lymphoproliferation involving the medial canthus and orbit has been reported, but may have represented orbital extension from primary cutaneous anaplastic large cell lymphoma of the eyelid. ${ }^{22}$ Interestingly, FISH in the patient with localized disease of the nasal cavity (case 1) demonstrated a rearrangement of the DUSP22-IRF4 locus on 6p25.3. Rearrangements of 6p25.3 are recurrent in ALK-negative anaplastic large cell lymphomas and are most common in primary cutaneous anaplastic large cell lymphoma, where they are seen in about $28 \%$ of cases. ${ }^{8,23-25}$ This finding is an additional feature shared between patients with primary mucosal CD30-positive T-cell lymphoproliferations and primary cutaneous cases. A 6p25.3 rearrangement also was seen in a patient with orbital disease (case 15) but unfortunately staging data were not available.

Two patients in our series had disease involving both mucosal sites and skin without evidence of disease elsewhere (eg, nodal, visceral or bone marrow). The skin processes were mycosis fungoides (case 8) and primary cutaneous anaplastic large cell lymphoma (case 9), with mucosal disease presenting in the nasal cavity and tongue, respectively. Occasional cases of mycosis fungoides ${ }^{26-28}$ and lymphomatoid papulosis ${ }^{16,21,29-32}$ have been reported to involve both cutaneous and mucosal sites, particularly the oral cavity. Some cases of lymphomatoid papulosis with oral involvement also showed genital involvement. ${ }^{21,31}$ Although comparative molecular studies were not performed in most cases, identical clonal rearrangements in the cutaneous and mucosal lesions have been reported in at least two patients. ${ }^{16,27}$ Adequate material for comparative studies was not available from the two cases in our series. The existence of patients with mucocutaneous disease suggests mucosal CD30-positive T-cell lymphoproliferations may be biologically similar to cutaneous cases, possibly reflecting molecular features contributing to epitheliotropism of the CD30positive T cells. ${ }^{33}$ Oral involvement in patients with cutaneous mycosis fungoides typically occurs late in the clinical course and is associated with poor prognosis. ${ }^{26}$ Our patient with mycosis fungoides 

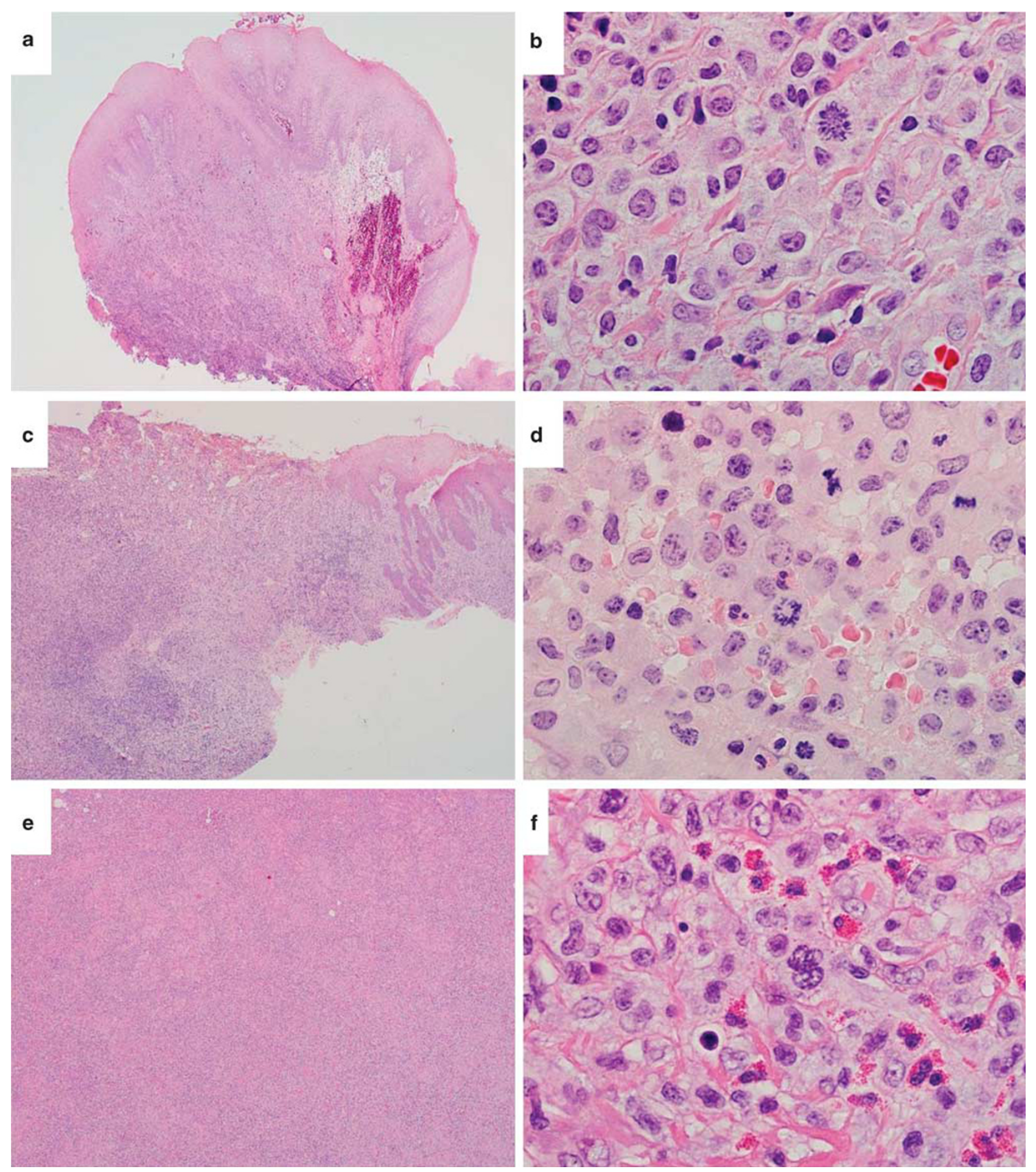

Figure 2 Mucosal CD30-positive T-cell lymphoproliferations with evidence of extramucosal disease. (a, b) Case 9. Biopsy of a nonulcerated, nodular tongue lesion from a 84-year-old male with history of primary cutaneous anaplastic large cell lymphoma shows a submucosal mononuclear cell infiltrate $(\mathbf{a}$; hematoxylin and eosin $(\mathrm{H} \& \mathrm{E}), \times 40)$. The lesion contains large, 'hallmark' cells without eosinophils $(\mathbf{b} ; \times 1000)$. (c, d) Case 10. Biopsy of a hard palate lesion from a 5-year-old male shows an extensive submucosal infiltrate (c; $\times 40$ ). There are large 'hallmark' cells and numerous eosinophils $(\mathbf{d} ; \times 1000)$. Phenotyping and staging revealed systemic ALK-positive anaplastic large cell lymphoma. (e, f) Case 11. Biopsy of an upper lip lesion from a 50-year-old female with a history of systemic ALKnegative anaplastic large cell lymphoma shows an extensive mononuclear cell infiltrate $(\mathbf{e} ; \times 40)$. The lesion contains large, 'hallmark' cells and numerous eosinophils $(\mathbf{f} ; \times 1000)$. $(\mathbf{g}-\mathbf{h})$ Case 13. Biopsy of an oral cavity mass from a 52 year-old male with systemic ALKnegative anaplastic large cell lymphoma shows an extensive submucosal infiltrate with overlying ulceration $(\mathrm{g} ; \times 40)$. High power shows numerous eosinophils $(\mathbf{h} ; \times 1000)$. 

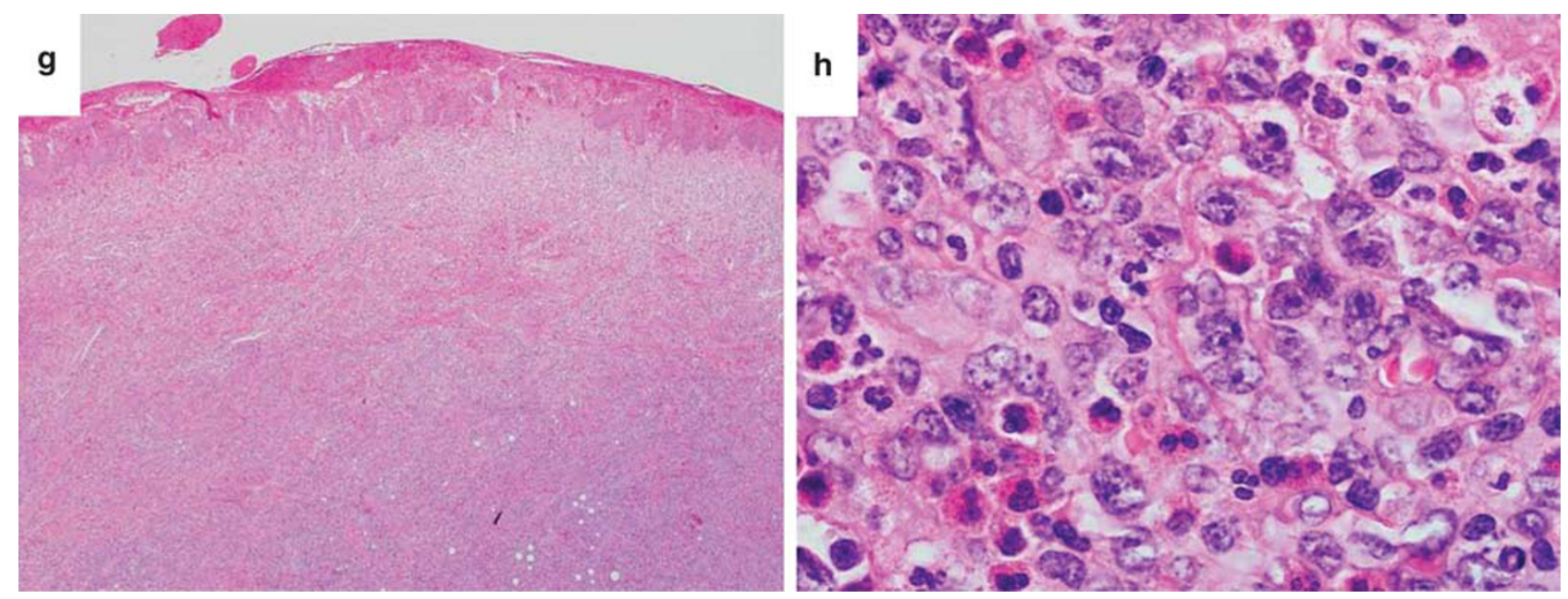

Figure 2 Continued.

(case 8) died of unrelated causes 19 months after presentation with disease in the nasal cavity, at which time he had persistent cutaneous disease but no evidence of mucosal disease. Therefore, we do not believe the mucosal disease represented transformed mycosis fungoides. Our patient with primary cutaneous anaplastic large cell lymphoma (case 9) received radiation therapy to both his cutaneous and mucosal lesions and was alive with no evidence of disease at last follow-up (66 months). We did not observe cases of lymphomatoid papulosis with a mucocutaneous pattern of distribution, but these have been reported to have the spontaneous regression and/or waxing and waning clinical course and overall favorable prognosis typical of that entity. ${ }^{21,29-31}$

Five of our cases had systemic anaplastic large cell lymphoma, which was ALK negative in four and ALK positive in one. Three of the five patients, all with ALK-negative anaplastic large cell lymphoma, died of anaplastic large cell lymphoma 1-48 months after presentation with mucosal disease. This mortality rate in patients with secondary mucosal lesions is similar to that reported in secondary cutaneous involvement by systemic anaplastic large cell lymphoma ${ }^{4}$ and also reflects the generally poor prognosis of systemic ALK-negative anaplastic large cell lymphoma (49\% 5-year overall survival) compared with either primary cutaneous anaplastic large cell lymphoma (90\% 5-year overall survival) or systemic ALK-positive anaplastic large cell lymphoma (70\% 5-year overall survival). ${ }^{5}$ Skin is a recognized secondary site of involvement by systemic ALKpositive and ALK-negative anaplastic large cell lymphomas, ${ }^{2,34}$ but secondary mucosal involvement is less well documented. Two systemic ALK-positive anaplastic large cell lymphomas have been reported that presented clinically with symptoms referable to involvement of the conjunctiva and orbit, respectively. ${ }^{35,36}$ The single case that was initially diagnosed on biopsy of the mucosal site in our series (case 10) also was ALK positive. This 5-year-old male presented with cervical lymphadenopathy unresponsive to antibiotics and a hard palate mass; biopsy of the palate lesion led to the diagnosis of ALK-positive anaplastic large cell lymphoma. The four systemic ALK-negative anaplastic large cell lymphomas in our series all presented with mucosal disease after their systemic disease had been diagnosed. Mucosal lesions in these patients had gross, histologic and phenotypic findings similar to lesions in patients without systemic disease. Ulceration was present in all evaluable cases, and eosinophils were prominent in two of five cases and occasionally present in one.

Taken together, our findings identify clinical and pathologic similarities between CD30-positive T-cell lymphoproliferations arising in mucosal sites and cutaneous CD30-positive T-cell lymphoproliferative disorders. First, both processes may represent either the primary and only site of disease or a manifestation of systemic anaplastic large cell lymphoma. As is the case for most cutaneous presentations of ALKpositive anaplastic large cell lymphoma, systemic disease was identified in our mucosal ALK-positive case. In contrast, we identified a 6 p25.3 rearrangement, most closely associated with primary cutaneous anaplastic large cell lymphoma, in a case with primary mucosal disease. The majority of mucosal cases shared morphologic and phenotypic features with both primary cutaneous anaplastic large cell lymphoma and systemic ALK-negative anaplastic large cell lymphoma, emphasizing the importance of a thorough history and clinical staging in patients with mucosal disease. Similar to primary cutaneous anaplastic large cell lymphoma, cases in which clinical staging indicated disease limited to the mucosa had a favorable prognosis. CD30-positive 
T-cell lymphoproliferations with similar features have been reported arising in association with breast implants. ${ }^{37}$ These lesions typically have localized disease and an indolent clinical course, although breast implants also can be involved in the setting of either systemic anaplastic large cell lymphoma or primary cutaneous anaplastic large cell lymphoma. ${ }^{38}$

Designating primary mucosal CD30-positive T-cell lymphoproliferations as ALK-negative anaplastic large cell lymphomas (or other peripheral T-cell lymphoma subtype) might lead to overtreatment as suggested previously. ${ }^{16,18}$ As shown in Table 2, some patients in our series with disease limited to the mucosa received systemic chemotherapy. It is not possible from our retrospective data to determine whether this therapy was beneficial, and consequently we cannot make treatment recommendations for these localized cases. Conversely, we would not classify our primary mucosal cases as traumatic ulcerative granuloma with stromal eosinophila, as they were not limited to the oral cavity and were not associated with known trauma. Traumatic ulcerative granuloma with stromal eosinophila (or eosinophilic ulcer of the oral mucosa) describes a specific reactive entity, but care should be taken to restrict use of this term to the proper clinical setting to avoid confusion and possibly understaging of patients with true T-cell neoplasms. The term traumatic eosinophilic granuloma appears less specific, as it has been used to describe various lesions, not all of which contain CD30-positive large T cells. ${ }^{16}$ Although neither the presence of cytologic atypia nor the demonstration of a clonal T-cell receptor gene rearrangement is sufficient for a diagnosis of lymphoma, ${ }^{19,39}$ our finding of occasional 6p25.3 rearrangements suggests a neoplastic rather than reactive process in at least some of our cases. Importantly, we show that systemic anaplastic large cell lymphomas may have mucosal findings overlapping those described in traumatic ulcerative granuloma with stromal eosinophila, including oral location, ulceration and eosinophils. Thus, we would recommend clinical staging for patients with mucosal CD30-positive T-cell lymphoproliferations with sheet-like growth of the large cells, an aberrant phenotype, extra-oral location or without antecedent trauma.

\section{Acknowledgements}

We thank Dr Elaine S Jaffe (Bethesda, MD, USA) for helpful comments on the paper. ALF is a Damon Runyon Clinical Investigator supported by the Damon Runyon Cancer Research Foundation (CI-48-09). This study was funded in part by the Division of Anatomic Pathology, Mayo Clinic, Rochester, MN, USA. The findings were presented in part at the 100th Annual Meeting of the United States and Canadian Academy of Pathology, 26 February-4 March 2011, San Antonio, TX, USA.

\section{Disclosure/conflict of interest}

The authors declare no conflict of interest.

\section{References}

1 Swerdlow S, Campo E, Harris N, et al. (eds). WHO Classification of Tumours of Haematopoietic and Lymphoid Tissues. International Agency for Research on Cancer: Lyon, France, 2008.

2 Mason DY, Harris NL, Delsol G, et al. Anaplastic large cell lymphoma, ALK-negative. In: Swerdlow S, Campo $\mathrm{E}$, Harris $\mathrm{N}$, et al. (eds). WHO Classification of Tumours of Haematopoietic and Lymphoid Tissues, 4th edn, Vol., International Agency for Research on Cancer: Lyon, France, 2008, pp 317-319.

3 Ralfkiaer E, Willemze R, Paulli M, et al. Primary cutaneous CD30-positive T-cell lymphoproliferative disorders. In: Swerdlow S, Campo E, Harris N, et al. (eds). WHO Classification of Tumours of Haematopoietic and Lymphoid Tissues, 4 edn, Vol., International Agency for Research on Cancer: Lyon, France, 2008, pp 300-301.

4 Bekkenk MW, Geelen FA, van Voorst Vader PC, et al. Primary and secondary cutaneous CD30(+) lymphoproliferative disorders: a report from the Dutch Cutaneous Lymphoma Group on the long-term follow-up data of 219 patients and guidelines for diagnosis and treatment. Blood 2000;95:3653-3661.

5 Savage KJ, Harris NL, Vose JM, et al. ALK- anaplastic large-cell lymphoma is clinically and immunophenotypically different from both ALK+ ALCL and peripheral T-cell lymphoma, not otherwise specified: report from the International Peripheral T-Cell Lymphoma Project. Blood 2008;111:5496-5504.

6 Kurtin PJ, Hobday KS, Ziesmer S, et al. Demonstration of distinct antigenic profiles of small B-cell lymphomas by paraffin section immunohistochemistry. Am J Clin Pathol 1999;112:319-329.

7 Morice WG, Katzmann JA, Pittelkow MR, et al. A comparison of morphologic features, flow cytometry, TCR-Vbeta analysis, and TCR-PCR in qualitative and quantitative assessment of peripheral blood involvement by Sezary syndrome. Am J Clin Pathol 2006;125: 364-374.

8 Feldman AL, Law M, Remstein ED, et al. Recurrent translocations involving the IRF4 oncogene locus in peripheral T-cell lymphomas. Leukemia 2009;23:574-580.

9 Benharroch D, Meguerian-Bedoyan Z, Lamant L, et al. ALK-positive lymphoma: a single disease with a broad spectrum of morphology. Blood 1998;91:2076-2084.

10 Isaacson PG, Chott A, Nakamura S, et al. Extranodal marginal zone lymphoma of mucosa-associated lymphoid tissue (MALT lymphoma). In: Swerdlow S, Campo E, Harris N, et al. (eds). WHO Classification of Tumours of Haematopoietic and Lymphoid Tissues, 4th edn, Vol. International Agency for Research on Cancer: Lyon, France, 2008, pp 214-217.

11 Elzay RP. Traumatic ulcerative granuloma with stromal eosinophilia (Riga-Fede's disease and traumatic eosinophilic granuloma). Oral Surg Oral Med Oral Pathol 1983;55:497-506.

12 el-Mofty SK, Swanson PE, Wick MR, et al. Eosinophilic ulcer of the oral mucosa. Report of 38 new cases with immunohistochemical observations. Oral Surg Oral Med Oral Pathol 1993;75:716-722. 
13 Mezei MM, Tron VA, Stewart WD, et al. Eosinophilic ulcer of the oral mucosa. J Am Acad Dermatol 1995;33:734-740.

14 Rosenberg A, Biesma DH, Sie-Go DM, et al. Primary extranodal CD3O-positive T-cell non-Hodgkins lymphoma of the oral mucosa. Report of two cases. Int J Oral Maxillofac Surg 1996;25:57-59.

15 Ficarra G, Prignano F, Romagnoli P. Traumatic eosinophilic granuloma of the oral mucosa: a CD30+(Ki-1) lymphoproliferative disorder? Oral Oncol 1997;33: 375-379.

16 Alobeid B, Pan LX, Milligan L, et al. Eosinophil-rich CD30+ lymphoproliferative disorder of the oral mucosa. A form of 'traumatic eosinophilic granuloma'. Am J Clin Pathol 2004;121:43-50.

17 Hirshberg A, Amariglio N, Akrish S, et al. Traumatic ulcerative granuloma with stromal eosinophilia: a reactive lesion of the oral mucosa. Am J Clin Pathol 2006;126:522-529.

18 Agarwal M, Shenjere P, Blewitt RW, et al. CD30positive T-cell lymphoproliferative disorder of the oral mucosa-an indolent lesion: report of 4 cases. Int J Surg Pathol 2008;16:286-290.

19 Salisbury CL, Budnick SD, Li S. T-cell receptor gene rearrangement and CD30 immunoreactivity in traumatic ulcerative granuloma with stromal eosinophilia of the oral cavity. Am J Clin Pathol 2009;132:722-727.

20 Segura S, Romero D, Mascaro Jr JM, et al. Eosinophilic ulcer of the oral mucosa: another histological simulator of $\mathrm{CD} 30+$ lymphoproliferative disorders. $\mathrm{Br} \mathrm{J}$ Dermatol 2006;155:460-463.

21 Allabert C, Esteve E, Joly P, et al. Atteinte muqueuse dans la papulose lymphomatoide: quatre cas. Ann Dermatol Venereol 2008;135:273-278.

22 Koreen IV, Cho RI, Frueh BR, et al. Primary cutaneous anaplastic large cell lymphoma of the medial canthus and orbit. Ophthal Plast Reconstr Surg 2009;25:63-65.

23 Pham-Ledard A, Prochazkova-Carlotti M, Laharanne E, et al. IRF4 gene rearrangements define a subgroup of CD30-positive cutaneous T-cell lymphoma: a study of 54 cases. J Invest Dermatol 2010;130:816-825.

24 Wada DA, Law ME, Hsi ED, et al. Specificity of IRF4 translocations for primary cutaneous anaplastic large cell lymphoma: a multicenter study of 204 skin biopsies. Mod Pathol 2011;24:596-605.

25 Feldman AL, Dogan A, Smith DI, et al. Discovery of recurrent $\mathrm{t}(6 ; 7)(\mathrm{p} 25.3 ; \mathrm{q} 32.3)$ translocations in ALKnegative anaplastic large cell lymphomas by massively-parallel genomic sequencing. Blood 2011;117: 915-919.

26 Sirois DA, Miller AS, Harwick RD, et al. Oral manifestations of cutaneous T-cell lymphoma. A report of eight cases. Oral Surg Oral Med Oral Pathol 1993; 75:700-705.
27 May SA, Jones D, Medeiros LJ, et al. Oral-cutaneous CD4-positive T-cell lymphoma: a study of two patients. Am J Dermatopathol 2007;29:62-67.

28 Gruson LM, Heller P, Hemmerdinger SA, et al. Mycosis fungoides involving the nasal mucosa. J Am Acad Dermatol 2007;56:S112-S114.

29 Sciubba J, Said-Al-Naief N, Fantasia J. Critical review of lymphomatoid papulosis of the oral cavity with case report. Oral Surg Oral Med Oral Pathol Oral Radiol Endod 2000;90:195-204.

30 Pujol RM, Muret MP, Bergua P, et al. Oral involvement in lymphomatoid papulosis. Report of two cases and review of the literature. Dermatology 2005;210:53-57.

31 Chimenti S, Fargnoli MC, Pacifico A, et al. Mucosal involvement in a patient with lymphomatoid papulosis. J Am Acad Dermatol 2001;44:339-341.

32 Kato N, Tomita Y, Yoshida K, et al. Involvement of the tongue by lymphomatoid papulosis. Am J Dermatopathol 1998;20:522-526.

33 van Kester MS, Tensen CP, Vermeer $\mathrm{MH}$, et al. Cutaneous anaplastic large cell lymphoma and peripheral T-cell lymphoma NOS show distinct chromosomal alterations and differential expression of chemokine receptors and apoptosis regulators. J Invest Dermatol 2010;130:563-575.

34 Delsol G, Falini B, Muller-Hermelink HK, et al. Anaplastic large cell lymphoma, ALK-positive. In: Swerdlow S, Campo E, Harris N, et al. (eds). WHO Classification of Tumours of Haematopoietic and Lymphoid Tissues, 4th edn, Vol. International Agency for Research on Cancer: Lyon, France, 2008, pp 312316.

35 Clarke B, Legodi E, Chrystal V, et al. Systemic anaplastic large cell lymphoma presenting with conjunctival involvement. Arch Ophthalmol 2003;121: $568-570$.

36 Sanka RK, Eagle Jr RC, Wojno TH, et al. Spectrum of CD30+ lymphoid proliferations in the eyelid lymphomatoid papulosis, cutaneous anaplastic large cell lymphoma, and anaplastic large cell lymphoma. Ophthalmology 2010;117:343-351.

37 Roden AC, Macon WR, Keeney GL, et al. Seromaassociated primary anaplastic large-cell lymphoma adjacent to breast implants: an indolent T-cell lymphoproliferative disorder. Mod Pathol 2008;21:455463.

38 Miranda RN, Lin L, Talwalkar SS, et al. Anaplastic large cell lymphoma involving the breast: a clinicopathologic study of 6 cases and review of the literature. Arch Pathol Lab Med 2009;133:1383-1390.

39 Cepeda LT, Pieretti M, Chapman SF, et al. CD30positive atypical lymphoid cells in common nonneoplastic cutaneous infiltrates rich in neutrophils and eosinophils. Am J Surg Pathol 2003;27:912-918. 